\title{
Hierarchy of Power System Computing Cloud and Its Application on On- line Parallel Analysis of Power Network
}

\author{
Yin ZHANG ${ }^{1}$ \& Jian GUO ${ }^{1}$ \& Yupei $\mathrm{JIA}^{1}$ \& Yansheng LANG ${ }^{1}$ \\ Jinghuai LIN² \& Zhengping CHEN ${ }^{2}$ \\ ${ }^{1}$ China Electric Power Research Institute, Beijing, China \\ ${ }^{2}$ State Grid Fujian Electric Power Co., Ltd. Fuzhou, China
}

\begin{abstract}
KEYWORD: cloud computing, power system, on-line analysis, parallel computing, distributed communication

ABSTRACT: In this paper, the online analysis framework based on cloud computing is presented, and the numerical calculation types of the power system is summarized. A collaborative computing model is presented on the basis of the analysis and comparison of parallel computing model and distributed computing model. This model is a parallel numerical model which support distributed communication, intermediate data transmission and unified monitoring. It realized the loose integration of the data cloud and the model cloud, achieves the requirements of the integrated computing. Finally, we apply the computing cloud architecture to state estimation and static contingency analysis which have the typical parallel and distributed computing mode. The practical performance can meet the requirements of the future scheduling model for online analysis and calculation.
\end{abstract}

\section{INTRODUCTION}

The fast development of smart grid promotes the construction of "Three Hua" UHV synchronous power network and the construction of the northwest and northeast power grid with large delivery end. The characteristics of the power network are changing from the regional mode to the global mode. The relation between power grids is also increasingly close, and new requirements for the integrated operation are put forward. The construction of "Big Operation" system will become the future development trend of smart grid. The integration of power grid characteristics requires the integration of coordinating and control, and it has higher technical requirements. The development of cloud computing model has the potential to solve the key problems of the integrated operation of smart grid. With the application of scheduling cloud in power system, the collaborative computing framework of device model, measurement data and online analysis is the need to be further studied.

In this paper, we put forward an online computing analysis framework based on cloud computing. The numerical calculation of power system is summarized, On the basis of analysis and comparison of parallel computing and distributed computing model, the collaborative computing model between the online computing and cloud of model and data is given. It provide theoretical guidance for the practical application of the dispatching cloud.

\section{HIERARCHY OF ON-LINE COMPUTING CLOUD}

Cloud computing is the product of the combination of the grid computing, parallel computing, network storage, virtualization, fault tolerance and load balancing. Its first aim is to give the user an Internet business model to provide on-demand computing services. It is also a new development direction of distributed computing (Fig. 1). Cloud computing changes the traditional application-oriented computing model. It achieves the transformation of the distributed computing model from "data centralization and computing distribution" to "data distribution and computation distribution". It highlights computational reliability and scalability. It can dynamically allocate computing resources at the peak and trough, and it can also improve the utilization of resources. 


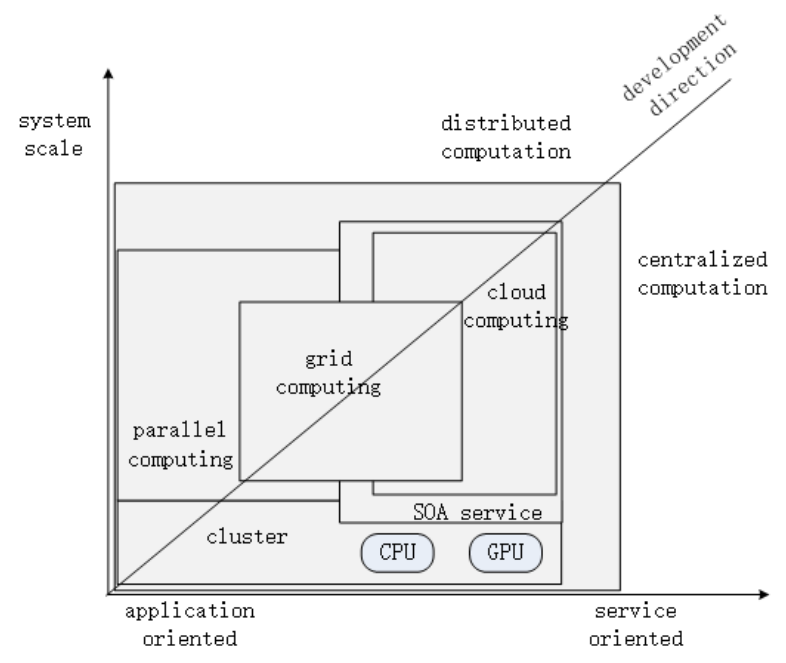

Figure 1. Cloud Computation Evolution.

Foreign power market is mature, the size of the grid and the development of the focus of the development is different from China. Taking into account the original intention of the cloud computing and its inherent security risks, foreign online analysis research based on cloud is in the state of "Wait and Watch". The domestic dispatching system and the development direction of the power grid are different from the foreign countries. Cloud computing is still in its initial stage in China, and it has some research on the management and analysis of massive data storage. Research is mainly on the historical data and power grid model of distributed storage management, formed a power grid model cloud and data cloud model. However, the research on the cloud computing is rarely published.

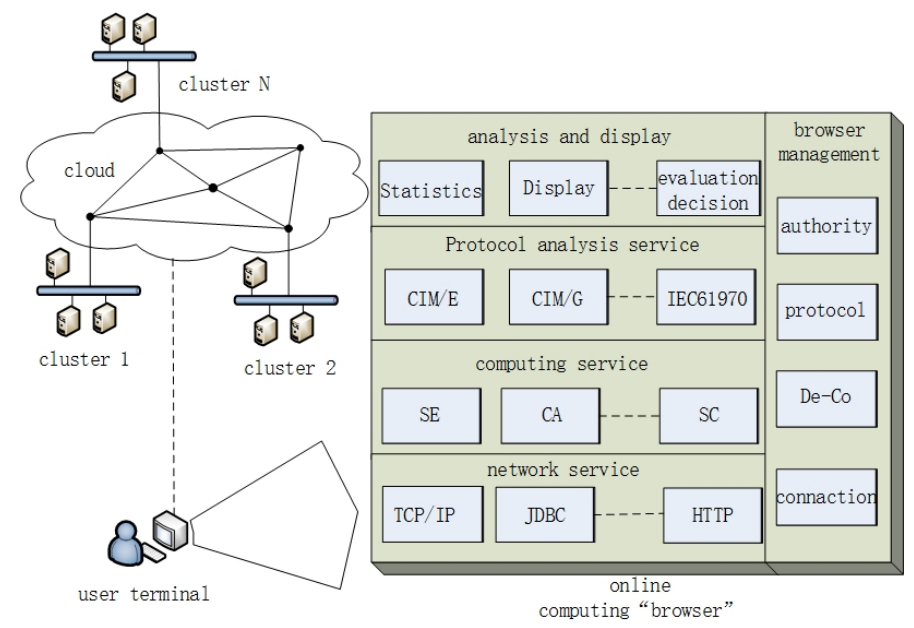

Figure 2. On-Line Computation "Browser".

The dispatching center obtains the real-time data through its exclusive dispatching communication network. At present, on-line analysis is carried out on the homogeneous cluster system. The safety partition, certification and quarantine measures have been taken to ensure data security and reliable communication. Power system online calculation based on unified online computing "browser" (Fig. 2) can play the advantages of parallel computing in local cluster and distributed computing in wide area.

The online analysis computing cloud involves parallel computing and distributed computing. Parallel computing mode is responsible for the parallel solution of the numerical method in order to improve the computing speed. Distributed computing model is responsible for data interaction with the model cloud and the measurement cloud, including management, computation process monitoring and fault 
recovery. Computing cloud use service oriented models to keep loose couple with model cloud and data cloud.

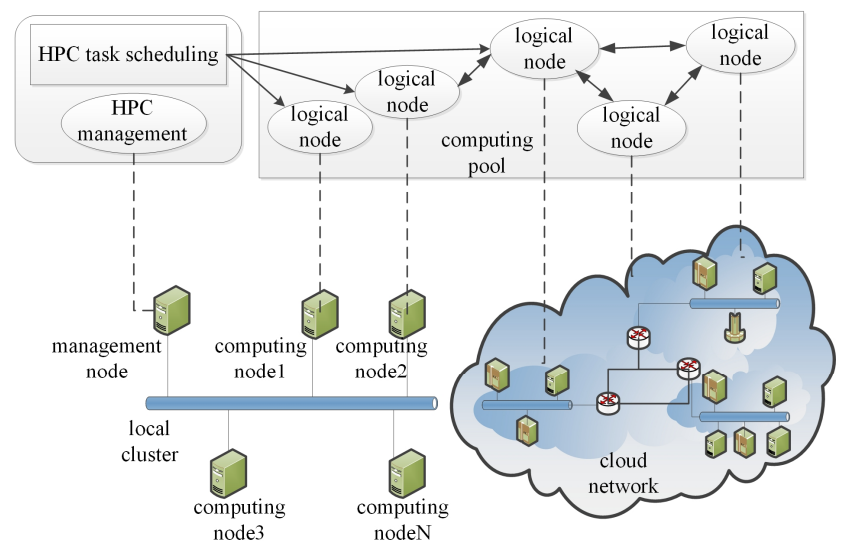

Figure 3. High Performance Computation Cloud.

\section{POWER SYSTEM NUMERICAL METHODS}

From the view of numerical analysis, power system parallel computing is divided into coarse-grained and fine-grained parallelization, which multi-tasks with little relevance, such as $\mathrm{N}-1$, short-circuit current calculation and dynamic security analysis. All involve iterative calculation of sparse linear equations and backward substitution operations, which belong to the fine-grained parallelized computing process. Types of power system parallel computing are summarizes in Table 1.

Table 1. Power System numerical analysis

\begin{tabular}{|l|l|l|}
\hline $\begin{array}{l}\text { Parallel gra- } \\
\text { nularity }\end{array}$ & $\begin{array}{l}\text { Calculation } \\
\text { type }\end{array}$ & $\begin{array}{l}\text { Numerical solu- } \\
\text { tions }\end{array}$ \\
\hline $\begin{array}{l}\text { Coarse-grain } \\
\text { computation }\end{array}$ & N-1 & $\begin{array}{l}\text { Repeated linear } \\
\text { equations }\end{array}$ \\
\cline { 2 - 3 } & $\begin{array}{l}\text { Short circuit } \\
\text { computation }\end{array}$ & $\begin{array}{l}\text { Repeated linear } \\
\text { equations }\end{array}$ \\
\cline { 2 - 3 } & $\begin{array}{l}\text { Transient } \\
\text { computation }\end{array}$ & $\begin{array}{l}\text { Differential/linear } \\
\text { equations }\end{array}$ \\
\cline { 2 - 3 } & $\begin{array}{l}\text { Optimal } \\
\text { power flow }\end{array}$ & $\begin{array}{l}\text { Linear equations } \\
\text { with constraints }\end{array}$ \\
\hline \multirow{2}{*}{$\begin{array}{l}\text { Fine-grain } \\
\text { computation }\end{array}$} & Power flow & $\begin{array}{l}\text { State esti- } \\
\text { linear equations }\end{array}$ \\
\cline { 2 - 3 } & mation & linear equations \\
\hline
\end{tabular}

While coarse-grained and fine-grained parallelization have differences in application type, they have dependence and relationships in calculation process, for example, $\mathrm{N}-1$ analysis revises node admittance matrix to do power flow calculation time by time, transient stability calculation constructs differential algebraic equations and do continuous power flow calculations to consider dynamic changes of generators and loads in the time domain, besides power flow or state estimation is the iterative solution of sparse linear equations in numerical process. To further enhance speed, while in the multi-task level coarse-grained parallel computing process, can do fine-grained parallel computing, the process shows in Figure 4. 


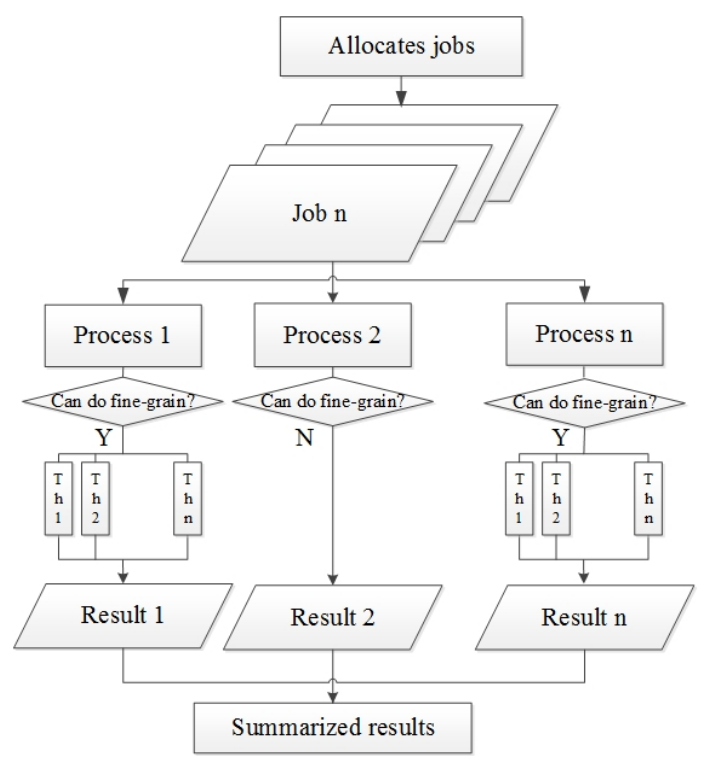

Figure 4. Computation process.

Coarse-grained tasks are decomposed to different processes to perform calculations simultaneously, the relations between each other is independently, and each has complete data and instructions memory space through distributed storage mode, so communication frequency is not high; each process first determines whether can do fine-grained parallelization, if yes, data is stored in shared mode and it generates several threads to execute tasks concurrently, which involve frequent data exchange and synchronization, if not, then process will calculate in serial.

\section{DISTRIBUTED COMPUTING AND DATA MANAGEMENT BASE ON AGENT}

Distributed computing based on cloud computing architecture is to achieve reliable communication support and calculation process unified monitoring. It needs to store the intermediate data in the calculation process, to recover the possible failure, and to ensure the quality of communication.Considering the multi-level scheduling management architecture, we use the hierarchical QoS proxy, which uses publish and subscribe push mode, to realize asynchronous distributed computing (Fig. 5).

HADC can provide end-to-end QoS properties such as fault tolerance, delivery rate, priority, redundancy and network security for a publish-subscribe middleware framework in general and status variables in particular, because the QoS agents have information about the network topology and network state of the domain that they administer. 


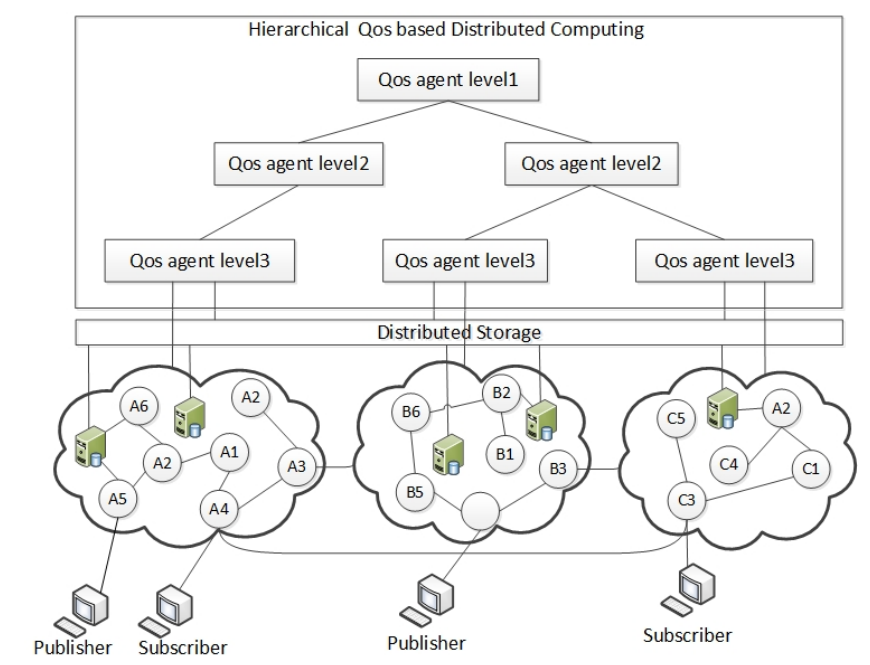

Figure.5 Hierarchical Architecture of Distributed Communication (HADC)

The main passive HADC entities are status variables and QoS policies, and the main active entities are publishers, subscribers, agent router and QoS agents, which are explained in more detail:

Status variable represents some device or phenomenon that has a state that changes over time. The sequence of states of a status variable is captured in status events, which are periodically published. Status events are encapsulated into status messages that flow through HADC communication infrastructure from publishers to subscribers. Each status variable has static attributes such as publisher name, status name, and rate, and dynamic attributes consisting of its current value and derived attributes such as its moving average, rate of change, moving average of the rate of change, and minimum and maximum values over a time interval.

Publishers are the sources of events in the publish-subscribe model. Publishers connect to the communication infrastructure through agent router. A publisher declares to its agent router each status variable that it will publish along with its QoS properties. After declaring a status variable, the publisher starts publishing status events with the QoS properties that it registered.

Agent routers make up the communication infrastructure. The agent routers are connected to each other through point-to-point or overlay links. They behave as smart routers, receiving and forwarding status messages. A group of agent routers in one administrative domain forms a cloud, which is controlled by a leaf QoS agent. Different clouds are connected with links between agent routers in the two clouds.

QoS policies are enforced by QoS agent. The policies affect admission control, security issues, fault tolerant strategies, etc.

QoS agents form a hierarchy of QoS management, with leaf QoS brokers at the lowest level, which manage a domain comprising a set of status routers, publishers and subscribers. Each leaf QoS agent has information about the agent routers' topology that it manages as well as information regarding the current network state of its domain. The upper-level QoS agents manage multiple clouds and are responsible for allocating and de-allocating inter-cloud subscriptions.

\section{STATE ESTIMATION AND STATIC CONTINGENCY ANALYSIS BASED ON COMPUTING CLOUD}

State estimation is the basis of advanced application of energy management system. To ensure accuracy and convergence, the second order state estimation is the necessary condition for the realization of self-healing power grid. To prevent cascading failures, static contingency analysis is not only required for N-1 analysis, but also N-x analysis, so the calculation is huge. At the same time, the closer the power grid is getting to the operating limit, the higher the requirements for safety analysis and calculation is. 
So in the computation cloud architecture, state estimation is based on data level parallelism. Static contingency analysis is based on task level parallelism and data level parallelism according to the different storage locations of the model and the data in the cloud (Fig. 6).

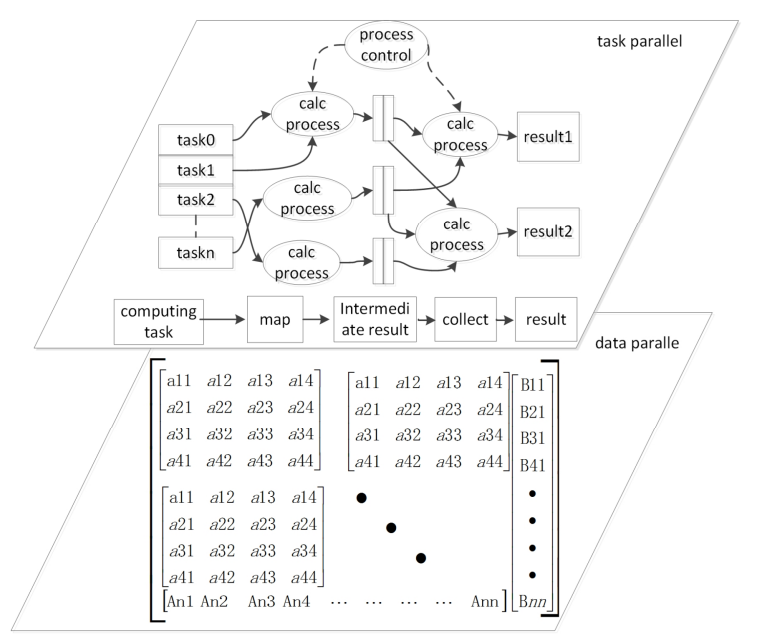

Figure 6. Parallelized Data and Task.

There is coupling between the data blocks in the data level parallelism, which requires frequent communication and data exchange. The correlation of multiple tasks in task level parallelism is week, and it only needs to summarize the results of the final analysis.

Parallel solutions of differential/algebraic equations in the calculations of contingency analysis and state estimation belong to data level parallelism. Fault calculation and margin assessment based on the specific operation flow section are task level parallel applications. Task level and data level parallelism are not unrelated to each other. To improve the calculation speed, task parallel computation can be performed at the same time the data level parallel computation is running.

\section{CONCLUSIONS}

Massive data are stored in distributed way and the grid is connected through Internet, which makes wide-area distributed and parallel computing suited for on-line analysis to improve computing performance. Cloud computing is a computing service abstracted from parallel and distributed computing, which provides a uniform interface to users.

Computing types of on-line analysis have different parallelism, such as state estimation is fine-grained parallelism and security analysis is coarse-grained parallelism. To fully exploit their parallelism, a hierarchical Qos agent of distributed communication which applied for publish-subscribe is proposed, guaranteed by Qos agent, Qos policies and agent router. The communication protocol of hierarchical agent of distributed computing to provide reliable computing service needs further research.

\section{ACKNOWLEDGEMENTS}

This work was financially supported by the Science and Technology Project of State Grid (Project No. DZ71-14-040).

\section{REFERENCES}

[1] Yousu, Chen, Zhenyu Huang,et al. 2012. Computational Challenges for Power System Operation, IEEE 45th Hawaii International Conference on System Science. 2141-2150.

[2] Above the clouds: A Berkeley View of Cloud Computing [OL]. http://www.eecs.berkeley.edu/Pubs/TechRpts/2009/EECS-2009-28.html, 2009. 
[3] Alvarado, F.L, Yu, D.C, Betancourt, R. 1990. Partitioned Sparse A-1 methods, IEEE Transactions on Power Systems, 5(2), 452-459.

[4] Lau,K. Tylavsky, D.J. Bose, A. 1991. Coarse grain scheduling in parallel triangular factorization and solution of power system matrices, IEEE Transactions on Power Systems, 6(2), 708-714.

[5] Naveen Cherukuri, Klara Nahrstedt. Cooperative Congestion Control in Power Grid Communication Networks, SmartGridComm11, 2011.

[6] Iosup, A. Osterman, S. Yigitbasi, M.N. et al. 2011. Performance Analysis of Cloud Computing Services for Many-Tasks Scientific Computing, IEEE Transaction on Parallel and Distributed Systems, 22(6), 931-945.

[7] Le Xie, Dae-Hyun Choi, SoummyaKar. Fully Distributed State Estimation for Wide-Area Monitoring Systems, IEEE Trans.Smart Grid.vol.3,no.3, pp.1154-1169, 2012

[8] Kawah Lau, Daniel J, Anjan Bose. Coarse grain scheduling in-parallel-triangular factorization and solution of power system matrices, IEEE Trans on Power Systems, vol.6, No.2, pp.708-714, 1991.

[9] K. Harald Gjermundrød, Ioanna Dionysiou, David Bakken, Carl Hauser and Anjan Bose. Flexible and Robust Status Dissemination Middleware for the Electric Power Grid, 2003 